\title{
Pancreatic cancer counterattack: MUC4 mediates Fas-independent apoptosis of antigen-specific cytotoxic $\mathbf{T}$ lymphocyte
}

\author{
YI ZHU ${ }^{1,2^{*}}$, JING-JING ZHANG ${ }^{1,2^{*}}$, WEN-BIAO LIANG ${ }^{3}$, RONG ZHU ${ }^{4}$, \\ BIN WANG $^{5}$, YI MIAO ${ }^{1,2}$ and ZE-KUAN XU ${ }^{1,2}$ \\ ${ }^{1}$ Department of General Surgery, First Affiliated Hospital, Nanjing Medical University, Nanjing; \\ ${ }^{2}$ Jiangsu Province Academy of Clinical Medicine, Institute of Tumor Biology, Nanjing, \\ Jiangsu 210029; ${ }^{3}$ Jiangsu Province Blood Center, Nanjing, Jiangsu 210042; ${ }^{4}$ Department of Pathology, \\ Shanghai Medical College, Fudan University, Shanghai 200032; ${ }^{5}$ Department of General Surgery, \\ The First Affiliated Hospital of Soochow University, Suzhou, Nanjing 215006, P.R. China
}

Received December 3, 2013; Accepted January 23, 2014

DOI: $10.3892 /$ or.2014.3016

\begin{abstract}
Tumor-associated MUC4 mucin has considerable potential as an immunotherapy target for pancreatic cancer. In previous studies, we developed dendritic cell (DC) vaccines which elicited MUC4 antigen-specific cytotoxic T lymphocyte (MS-CTL) response against tumor cells in vitro. Due to the observation that MS-CTL apoptotic rate increased significantly when co-cultured with $\mathrm{MUC}^{+}$tumor cells compared with $\mathrm{T} 2$ cells, we investigated whether high expression levels of MUC4 in pancreatic cancer cells would have an effect on the significant increase of apoptosis rate of MS-CTLs. First, the adverse influence of regulatory $\mathrm{T}$ cells (Tregs) was eliminated by $\mathrm{CD} 8^{+}$ T lymphocyte sorting before the induction of MS-CTLs. Then, we constructed clonal MUC4-knockdown HPAC pancreatic cancer sublines with different MUC4 expression for co-incubation system. By utilizing appropriate control to rule out the possible apoptosis-induced pathway of intrinsic activated cell-autonomous death (ACAD) and analogous antigendependent apoptosis of CTL (ADAC) in our study system, further analysis of the effect of MUC4 membrane-expression, supernatants and blockade of CTL surface Fas receptor on MS-CTL apoptosis was carried out. The results demonstrated that the level of MUC4 membrane expression strongly positively correlated with MS-CTL apoptosis and the influence of
\end{abstract}

Correspondence to: Professor Ze-Kuan Xu or Professor Yi Miao, Department of General Surgery, First Affiliated Hospital, Nanjing Medical University, 300 Guangzhou Road, Nanjing, Jiangsu 210029, P.R. China

E-mail: xuzekuan@njmu.edu.cn

E-mail: miaoyi@njmu.edu.cn

\section{${ }^{*}$ Contributed equally}

Key words: apoptosis, cytotoxic, MUC4, pancreatic neoplasms, T lymphocytes supernatants and Fas-blockade did not significantly correlate with MS-CTL apoptosis. This evidence suggested that there may be a novel counterattack pathway of pancreatic cancer cells, which is a MUC4-mediated, cell contact-dependent and Fas-independent process, to induce CTL apoptosis. Therefore, further exploration and understanding of the potential counterattack mechanisms is beneficial to enhance the efficacy of MUC4 specific tumor vaccines.

\section{Introduction}

It is well known that pancreatic cancer is a lethal disease that is difficult to treat. Surgical resection remains the only potential curative approach to a small proportion in early stage (1). There is a need for the continual development of multimodality treatments including immunotherapy to improve patient prognosis (2).

As a member of the transmembrane mucin family $(3,4)$, MUC4 is considered to have considerable potential as an immunotherapy target for pancreatic cancer $(5,6)$. MUC4, in particular, is aberrantly expressed in pancreatic ductal adenocarcinoma (PDAC) and precancerous pancreatic intraepithelial neoplasias (PanINs), but not in benign or normal parts of pancreas (7-9). The level of MUC4 expression correlates significantly with a poor prognosis of PDAC $(9,10)$. Previous studies suggested that cancer cells could use MUC4 for invasion, metastasis and resistance to chemotherapy (11-14). MUC4 antigen-pulsed vaccines are promising to eradicate focus in early stage and eliminate the minimal residual lesion remaining after surgical resection with minimizing damage to normal cells. Thus, the development in MUC4-antigen related vaccines is favorable for prevention of tumor recurrence and the approaches posses safety and tolerability with less substantial toxicity.

In our previous studies (15-17), we developed MUC4antigen related dendritic cell (DC) vaccines which elicited MUC4 antigen-specific cytotoxic T lymphocyte (MS-CTL) response against MUC4-expressing tumor cells in vitro. 
During examination of the cytotoxic activity of the vaccine in vitro, we observed the apoptosis of CTLs and noted that the apoptotic rate of CTLs co-cultured with $\mathrm{MUC4}^{+}$tumor cells was significantly increased compared to those co-cultured with T2 cells of pulsed MUC4-epitope- peptide, suggesting MUC4+ tumor cells could counterattack MS-CTLs via some pathway during the cytolytic process.

MUC4-bearing tumor cells lose their polarity, allowing MUC4 to be uniformly expressed all over the cell surface (18). With a larger size (ranges between 1.1 and $2.1 \mu \mathrm{m}$, depending on the length of the central tandem repeat domain) above the cell surface, MUC4 could mask most cell surface molecules (not exceeding a length of $35 \mathrm{~nm}$ ) and interrupt their functions on cell-cell interactions (18). On the other hand, the aberrant overexpression or effusion of MUC4 mucin, including distinct types protein (secreted or membrane-associated forms), confers on tumor cells potential ligands for interaction with other receptors at the cell surface, and they might inactivate immune effector cells through receptor-ligand interactions (19). Thus, we postulated that MUC4 might contribute to the enhanced apoptotic rate of MS-CTLs during the killing process.

In the present study, we first isolated pure $\mathrm{CD} 8^{+} \mathrm{T}$ cells before the induction and amplification of MS-CTLs to eliminate the killing influence of Tregs. Secondly, we fixed basic parameter and defined precise gating template for apoptosis measurements by flow cytometry in order to investigate the nature of the apoptosis of MS-CTLs in co-incubation system. Thirdly, we repeated comparative analysis of the apoptosis rate of MS-CTLs incubated with $\mathrm{MUC4}^{+}$tumor cells and pulsed-peptide T2 cells to confirm the previous observations. Then, we addressed whether high levels of MUC4 by pancreatic cancer cells would have an effect on the significant increase of apoptosis rate of MS-CTLs: i) clonal MUC4knockdown HPAC sublines with different MUC4 expression were selected for co-incubation system and further analyses were performed; ii) the total apoptosis-induced effects of supernatants on MS-CTLs were compared among distinct co-cultured groups; iii) analyses of the blocking effect of Fas on MS-CTL surface were applied to further elucidate whether the Fas-FasL pathway might be involved in MS-CTL apoptosis in co-incubation system.

\section{Materials and methods}

Cell lines, cytokines, culture medium, epitope peptide. T2 cells (HLA-A2+/MUC4-, TAP deficient) were a generous gift from Weifeng Chen (Immunity Department, Peking University). The three human HLA-A2 ${ }^{+} / \mathrm{MUC}^{+}{ }^{+}$tumor cell lines were obtained from the American Type Culture Collection (ATCC; Manassas, VA, USA), including HCT 116 (colorectal carcinoma), CFPAC-1 and HPAC (pancreatic adenocarcinoma). HCT 116 cells were grown in McCoy's-5A medium (SigmaAldrich, St. Louis, MO, USA). Other lines were cultured in DMEM (Gibco, Auckland, New Zealand), supplemented with $10 \%$ heat-inactivated fetal calf serum (Gibco, Carlsbad, CA, USA), $2 \mathrm{mM}$ glutamine, $100 \mathrm{U} / \mathrm{ml}$ penicillin, $100 \mathrm{mg} / \mathrm{ml}$ streptomycin at $37^{\circ} \mathrm{C}$ in $5 \% \mathrm{CO}_{2}$.

Recombinant cytokines of human GM-CSF, IL-4, TNF- $\alpha$, IL-2 were purchased from PeproTech (Rocky Hill, NJ, USA).
The HLA-A2-restricted and MUC4-specific CTL epitope peptides (LLGVGTFVV) were synthesized by Shanghai Sangon Biological Engineering Technology (Shanghai, China).

$H L A-A 2^{+}$volunteers. Three healthy HLA-A $2^{+}$donors (male, average age 24-30 years) from Jiangsu Provincial Blood Center (Nanjing, China) were screened from 45 volunteers, and the verification of HLA-A2 subtype was performed as previously described $(16,20)$. Samples were collected after obtaining patient informed consent and the present study was approved by the Ethics Committees of The First Affiliated Hospital of Nanjing Medical University (reference no: 2011-SRFA-100).

Induction of human HLA-A2-restricted and MUC4-specific $C T L$. Peripheral blood mononuclear cells (PBMCs) were isolated from buffy coats with Ficoll-Hypaque (TBD Science, Tianjin, China), as previously described (16). CD8 ${ }^{+} \mathrm{T}$ lymphocytes in the PBMCs separated from the blood samples were isolated by CD8 Dynabeads (Daynal Biotech, USA), according to the manufacturer's instructions. The isolated $\mathrm{CD}^{+} \mathrm{T}$ cells were analyzed by flow cytometry with anti-CD8-FITC antibodies (BD Pharmingen, San Jose, CA, USA).

DCs were induced by IL-4 and GM-CSF cocktail as previously described (16). On day 5, 1,000 U/ml of tumor necrosis factor- $\alpha$ (TNF- $\alpha)$ was added to induce final maturation. After 7 days of culture, DCs were harvested and were confirmed with mature DC-specific phenotype by FACS. Matured DCs $\left(1 \times 10^{5}\right.$ cells/well) were pulsed with $20 \mu \mathrm{g} / \mathrm{ml}$ epitope peptide (LLGVGTFVV) for $2 \mathrm{~h}$, washed, and irradiated by Co60 (2 Gy/min for $15 \mathrm{~min}$ ). The irradiated, peptide-loaded DCs were mixed with $1 \times 10^{6}$ cells/well $\mathrm{CD}^{+} \mathrm{T}$ cells at a final $\mathrm{DC}$ : $\mathrm{CD}^{+} \mathrm{T}$ cell ratio of 1:10. The co-cultures were seeded in U-bottom 96 -well plates and incubated for 3 days at $37^{\circ} \mathrm{C}, 5 \%$ $\mathrm{CO}_{2}$. IL-2 $(10 \mathrm{U} / \mathrm{ml})$ was added on day 4 and the same amount of loaded DCs was added on day 7 and day 10. On day 14, cells were harvested as MS-CTLs and used for subsequent MS-CTL/tumor cell interaction experiments.

Selection of clonal MUC4-knockdown HPAC sublines with different MUC4 expression

Construction of clonal MUC4-knockdown HPAC sublines. The target DNA sequences corresponding to MUC4-shRNA were previously reported $(21,22)$. The selected sequences are present in all splice variants of MUC4 characterized thus far, as follows: forward oligo: 5'-ccggAACGCAAGCATCGG ACTTCACctcgagGTGAAGTCCGATGCTTGCGTTtttttg-3'; reverse oligo: 5'-aattcaaaaAACGCAAGCATCGGACTTC ACctcgagGTGAAGTCCGATGCTTGCGTT-3', with AgeI and $E c o$ RI restriction enzyme sites (5' and $3^{\prime}$ ends, respectively). The target DNA was ligated to the pLKO.1-TRC cloning vector (23) (Addgene ID \#10879), which was supplied by PlusGene Center of Nanjing Medical University (Nanjing, China) and verified by sequencing. The verified recombinant vector plasmid (pLKO.1/MUC4-shRNA), the packaging plasmid $\mathrm{p}^{\Delta} 8.2$ and $\mathrm{pVSV}-\mathrm{G}$ were co-transfected into $293 \mathrm{~T}$ cells by Lipofectamine $^{\mathrm{TM}} 2000$ reagent (Invitrogen, Carlsbad, CA, USA). The supernatant of the cultured 293T cells was collected to infect the HPAC cells. The pLKO.1-scramble shRNA vector (Addgene ID \#1864; negative control vector containing scrambled shRNA insert) was used to package virus and infect 
HPACs as control. Stable clones were then selected in a medium containing puromycin ( $5 \mu \mathrm{g} / \mathrm{ml}$; Sigma). MUC4 expression in the derived sublines was confirmed via real-time RT-PCR, western blot analysis and flow cytometry.

\section{Identification of clonal MUC4-knockdown HPAC sublines.}

Quantitative real-time PCR. Total RNA was extracted from the selected MUC4-knockdown HPAC sublines with TRIzol reagent (Invitrogen). The reverse transcription using iScript cDNA synthesis kit (Bio-Rad Laboratories, Hercules, CA, USA) and quantitative real-time PCR using TaqMan Gene Expression assays (Applied Biosystems, Foster City, CA, USA) were performed as previously described (9). Each quantification PCR was performed in triplicate. The mRNA concentration was defined as the ratio of target mRNA copies relative to GAPDH mRNA copies.

Western blot analysis. The HPAC-derived clones were processed for protein extraction and western blotting using standard procedures. Cell lysates were prepared as previously described (24). Protein concentrations were determined by using the Bradford assay and proteins $(20 \mu \mathrm{g} /$ lane $)$ were resolved on $4-20 \%$ Mini-Protean ${ }^{\circledR}$ TGX $^{\text {TM }}$ precast gels (\#456-1093; Bio-Rad Laboratories). Resolved proteins were transferred onto the polyvinylidene difluoride (PVDF) membrane and blocked in 5\% non-fat milk in PBS for $2 \mathrm{~h}$ and subjected to the standard immunodetection procedure using specific antibodies. The following primary antibodies were used for labeling of the membranes: $1 \mu \mathrm{g} / \mathrm{ml}$ anti-MUC4 mouse monoclonal antibody (ab60720; Abcam, Cambridge, UK) for MUC4 immunodetection; 1:1,000-diluted antiGAPDH mouse monoclonal antibody (AG019; Beyotime Institute of Biotechnology, Haimen, China) for GAPDH immunodetection. The membranes were incubated at $4^{\circ} \mathrm{C}$ overnight followed by six 10 -min washes in TBST [ $50 \mathrm{mmol} / 1$ Tris- $\mathrm{HCl}$ (pH 7.4), $150 \mathrm{mmol} / \mathrm{l} \mathrm{NaCl}$, and $0.05 \%$ Tween20]. Membranes were then incubated with 1:2,000-diluted horseradish peroxidase (HRP)-labeled goat anti-mouse IgG (A0216; Beyotime) for $1 \mathrm{~h}$ at room temperature followed by six 10-min washes with TBST. The blots were developed with an enhanced chemiluminescence kit (Amersham Biosciences, Freiburg, Germany). PageRuler ${ }^{\mathrm{TM}}$ Plus Prestained Protein Ladder (\#26619/SM1811, 10-250 kD; Fermentas) were used as internal molecular weight standards. Each blot was repeated three times. The value of optical density (OD) was measured by the software (Quantity One v462; Bio-Rad Laboratories) and the relative protein expression levels of MUC4 were calculated with the following ratio: (OD value of MUC4 - OD value of background) / (OD value of GAPDH - OD value of background).

FACS analysis of surface expression of MUC4. Cells $\left(1 \times 10^{6}\right)$ were suspended in $100 \mu \mathrm{l}$ of PBS containing $1 \%$ BSA and incubated on ice for $30 \mathrm{~min}$ with $5 \mu \mathrm{g} / \mathrm{ml}$ monoclonal antiMUC4 (clone 1G8) antibodies (mouse IgG1; Invitrogen). After washing twice with $2 \mathrm{ml}$ of cold PBS, the cells were incubated on ice for $30 \mathrm{~min}$ with 1:1,000 diluted fluorescein isothiocyanate (FITC)-conjugated goat anti-mouse IgG antibody (eBiosciences), washed twice with PBS, and fixed in 1\% paraformaldehyde and, finally, 10,000 gated events were analyzed using a FACSCalibur flow cytometer (BD Biosciences). Each measurement was repeated three times. Geometric mean fluo- rescence intensity (GMFI) values of equal quantity cells only labeled primary antibodies were calculated as background values. GMFI values of equal quantity cells only labeled second antibodies were calculated as negative control (NC). Fluorescence index (FI) was calculated with the following formula: $\mathrm{FI}=($ GMFI sample - GMFI background $) /$ GMFI background.

\section{$M S$-CTL/tumor-cell co-incubation experiments}

Fixed basic co-incubation condition and standardizing measurements. Co-incubated system with basic condition and fixed parameter: MS-CTLs suspended in 10\% FCS RPMI-1640 medium were seeded into a round-bottomed 96-well plate at $5 \times 10^{5}$ cells $/ 100 \mu \mathrm{l} /$ well in triplicate. The prepared target cells were added into the corresponding wells at $1 \times 10^{5}$ cells $/ 100 \mu 1 /$ well to make the effector/target ratios equal 5:1, respectively. At the same time 10\% FCS RPMI-1640 medium without target cells was added into MS-CTLs suspended at a final volume of $200 \mu 1 /$ well as background control. The plates were spinned at $1,000 \mathrm{rpm}$ for $10 \mathrm{~min}$ and then incubated for $4 \mathrm{~h}$ at $37^{\circ} \mathrm{C}, 5 \%$ $\mathrm{CO}_{2}$, the same as the standard procedures of ${ }^{51} \mathrm{Cr}$ release assay (cytotoxicity assays) (25). After incubation, the plates were spinned again at $1,000 \mathrm{rpm}$ for $20 \mathrm{~min}$; $100 \mu \mathrm{l}$ supernatant were harvested for subsequent tests and $100 \mu \mathrm{l}$ co-incubated cells were harvested for the following apoptosis measurements of CTLs.

FACS-apoptosis measurements of CTLs from the co-incubated system: the co-incubated cells at a volume of $100 \mu \mathrm{l}$ were harvested into tube and labeled with $5 \mu \mathrm{l}$ of anti-CD8-APC antibodies (Invitrogen) for $20 \mathrm{~min}$ at room temperature, protected from light. Then, cells were resuspended in buffer and were stained with an Annexin V-FITC kit (Invitrogen Systems). Cell suspensions were evaluated using a FACSCalibur flow cytometer (BD Biosciences) within $1 \mathrm{~h}$ and the results were analyzed using CellQuest version 3.1 software (BD Biosciences). At least 10,000 events per sample were collected with debris and aggregates excluded using low forward and orthogonal light scatter values. Compensations were established using single color controls. The gates for CD8 positive cells were set to calculate $\mathrm{CTL}\left(\mathrm{CD}^{+}\right)$apoptosis. Cells that stained positively with Annexin $\mathrm{V}$ were considered to be apoptotic. Fig. 1C shows that CTLs were sorted out easily from co-incubated cells by CD8-APC positive gate and were calculated accurately.

Co-incubation experiments. A series of experiments was carried out based on basic condition (see above) as follows: i) the apoptosis rates of MS-CTLs incubated with MUC4 ${ }^{+}$ tumor cells (HCT 116, CFPAC-1 and HPAC) and pulsedpeptide T2 cells were compared to confirm the previous observations; ii) clonal MUC4-knockdown HPAC sublines with different MUC4 expression were selected as target cells and further MS-CTL apoptosis was examined; iii) the harvested supernatant of distinct groups, the same as ii), were used to incubate MS-CTLs for $4 \mathrm{~h}$ and following apoptosis measurement for the analysis of the influence of supernatant; iv) effect of blockade of Fas on MS-CTL surface. We hypothesized that the Fas-FasL pathway might contribute to MS-CTL apoptosis as one of the effect factors. Before co-incubating with target cells, also the same as ii), MS-CTLs were cultured for $1 \mathrm{~h}$ at $37^{\circ} \mathrm{C}$ in the presence of antagonistic mouse anti-human 

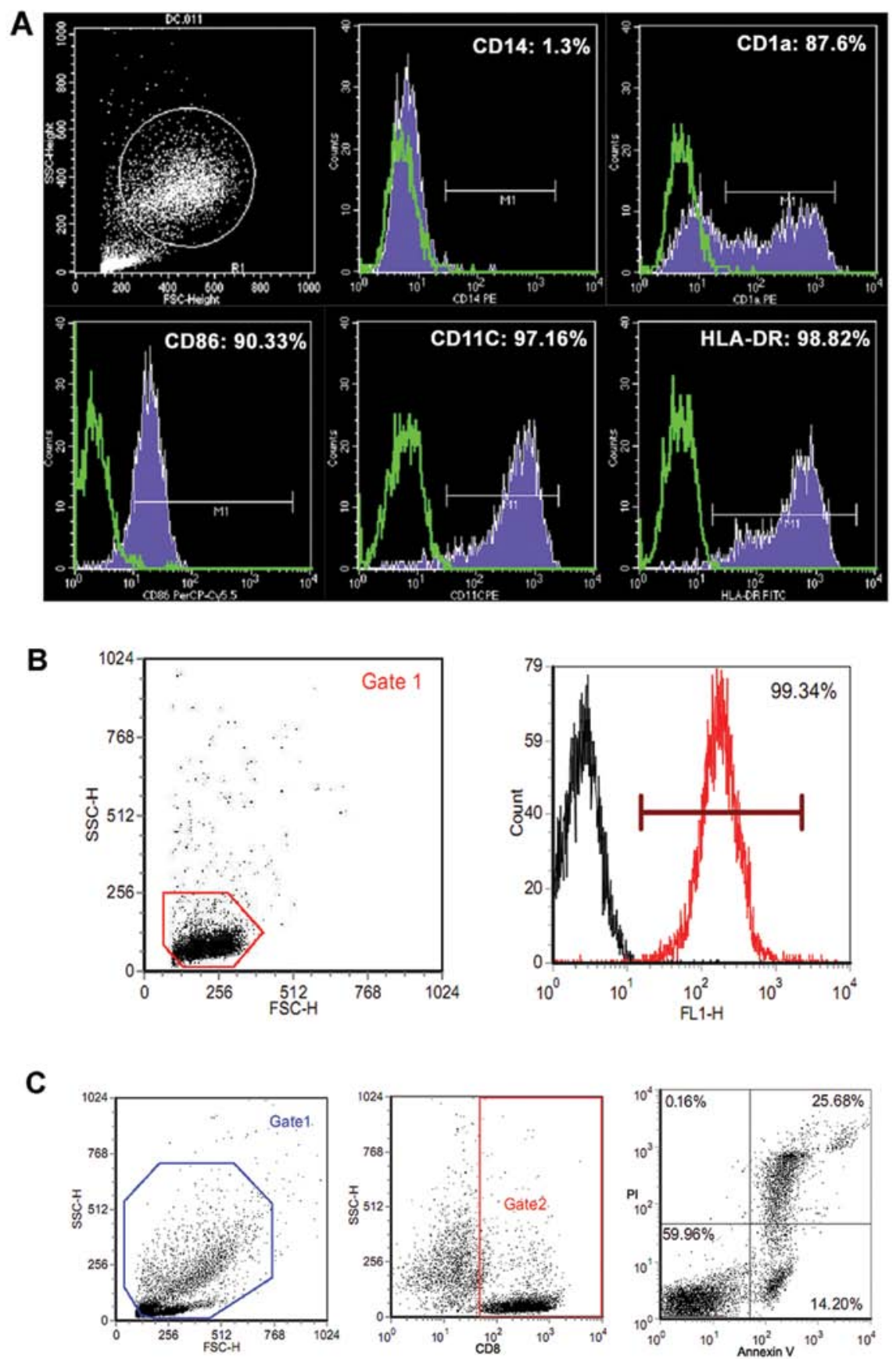

Figure 1. Representative templates of FACS analysis. (A) Phenotypic markers of mature dendritic cells; (B) purification of CD8 ${ }^{+}$T cells; (C) FACS apoptosis analysis of $\mathrm{CD}^{+}$-CTLs in a co-incubated system.

Fas, ZB4 (Beckman Coulter, Brea, CA, USA; $2 \mu \mathrm{g} / \mathrm{ml}$ ). The effective blocking dose was defined according to lymphocyte counting using tetrazolium bromide (MTT). After incubation, cells were collected for the detection of apoptosis.

Statistical analysis. Results are expressed as means \pm standard error (SEM). Data were analyzed using one-way analysis of variance between groups (ANOVA). Correlations between CTL apoptosis rates and determined factors were analyzed using linear regression analysis. The variations in blocking effect of Fas on CTL surface were analyzed by 2-way ANOVA. Statistical analysis was performed with SPSS 17.0 software. Differences were considered statistically significant at $\mathrm{P}<0.05$.

\section{Results}

Generation of mature DCs and MS-CTLs. After 7 days of culture in medium containing GM-CSF, IL-4 and TNF- $\alpha$, FACS analysis showed that DCs from blood samples of three HLA-A*0201 volunteers displayed typical mature phenotypic characteristics. The frequency of expression of the phenotypic markers CD14, CD86, CD1a, CD11c and HLA-DR was $1.32 \pm 0.12,89.13 \pm 3.76,90.24 \pm 8.63,97.32 \pm 1.23$ and $96.55 \pm 2.59 \%$, respectively (one representative FACS histogram is depicted in Fig. 1A). $\mathrm{CD}^{+} \mathrm{T}$ cells (purities $>99 \%$, as shown in Fig. 1B) were induced into human HLA-A2-restricted and MUC4-specific CTLs by co-culture with epitope peptide- 


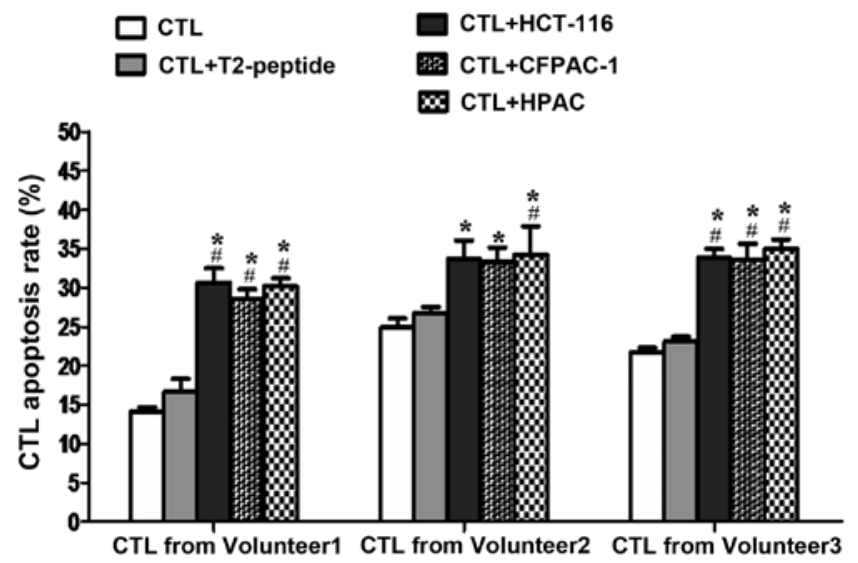

Figure 2. MS-CTL apoptosis rate increases significantly when co-cultured with $\mathrm{MUC}^{+}$tumor cells compared to the control. Effector and target cells with the ratios of 5:1 were co-incubated in 96 -well plates for $4 \mathrm{~h} .{ }^{*} \mathrm{P}<0.05$ in comparison with background control (CTLs without target cells added), ${ }^{\text {}} \mathrm{P}<0.05$ in comparison with pulsed-peptide T2 cells group. Error bars indicate the means \pm SEM of three determinations. One-way ANOVA plus Tukey's post hoc comparison was used to calculate the significant difference.

loaded DCs. The specific cytotoxic activity of MS-CTLs is consistent with our previous reports (16) (data not shown).

The apoptosis rate of MS-CTLs increases significantly when co-cultured with MUC4+ $4^{+}$tumor cells compared to the background control. Induction of MS-CTLs using blood sample from volunteer 1; compared to the background control group (CTLs without target cells added) or T2 cell group, the apoptosis rate of MS-CTLs in MUC4+ $4^{+}$tumor cell (HCT 116, CFPAC-1 and HPAC) groups increased significantly, $\mathrm{P}<0.05$, respectively (Fig. 2). Using blood samples from volunteers 2 and 3, the changing trend of MS-CTL apoptosis rate was similar, although the changing extent had a slight difference (Fig. 2).

Selection of MUC4-shRNA HPAC sublines with different $M U C 4$ expression. Seven stably transfected clones were identified via real-time RT-PCR. The expression level of MUC4 normalized to that of GAPDH is shown in Fig. 3A. Compared to HPAC (100\%), the MUC4 expression levels were 96.23 \pm 19.76 , $20.30 \pm 1.05,67.59 \pm 7.16,75.49 \pm 9.17,6.85 \pm 0.17,13.07 \pm 3.62$ and $43.35 \pm 3.15 \%$ in the stably transfected empty vector clone of HPAC (HPAC-EV), six stably transfected MUC4-shRNA clones of HPAC, i.e. HPAC-sh-\#1-6, respectively.

Three clones, i.e. HPAC-sh-\#3, HPAC-sh-\#4 and HPACsh-\#6, with a wide range of different MUC4 expression levels were selected for subsequent confirmation via western blot analysis and flow cytometry. Fig. 3B shows that the relative protein expression levels of MUC4 (vs. GAPDH) were $0.68 \pm 0.10,0.66 \pm 0.04,0.40 \pm 0.03,-0.0008 \pm 0.03$ and $0.21 \pm 0.01$ in HPAC, HPAC-EV, HPAC-sh-\#3, HPAC-sh-\#4 and HPACsh-\#6, respectively.

Cell membrane surface expression of MUC4 was determined by FACS analysis. Fig. 3C shows that the fluorescence index (FI) on behalf of relative surface expression levels of MUC4 was $54.40 \pm 1.32,46.32 \pm 1.53,17.56 \pm 0.69,0.97 \pm 0.09$, $8.61 \pm 0.51$ and $0.10 \pm 0.01$ in HPAC, HPAC-EV, HPAC-sh-\#3, HPAC-sh-\#4, HPAC-sh-\#6 and NC (negative control), respec-

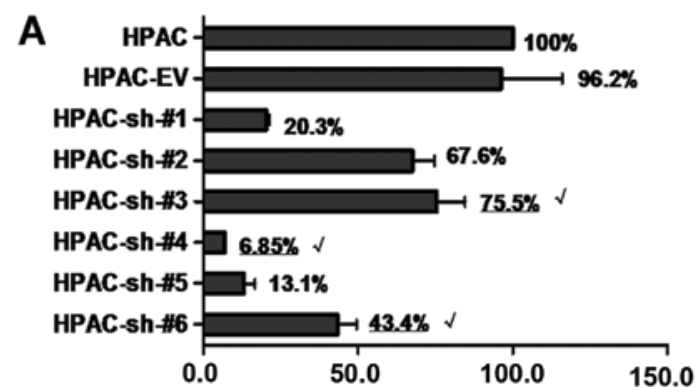

MUC4 mRNA expression (VS.HPAC) in stably transfected clones
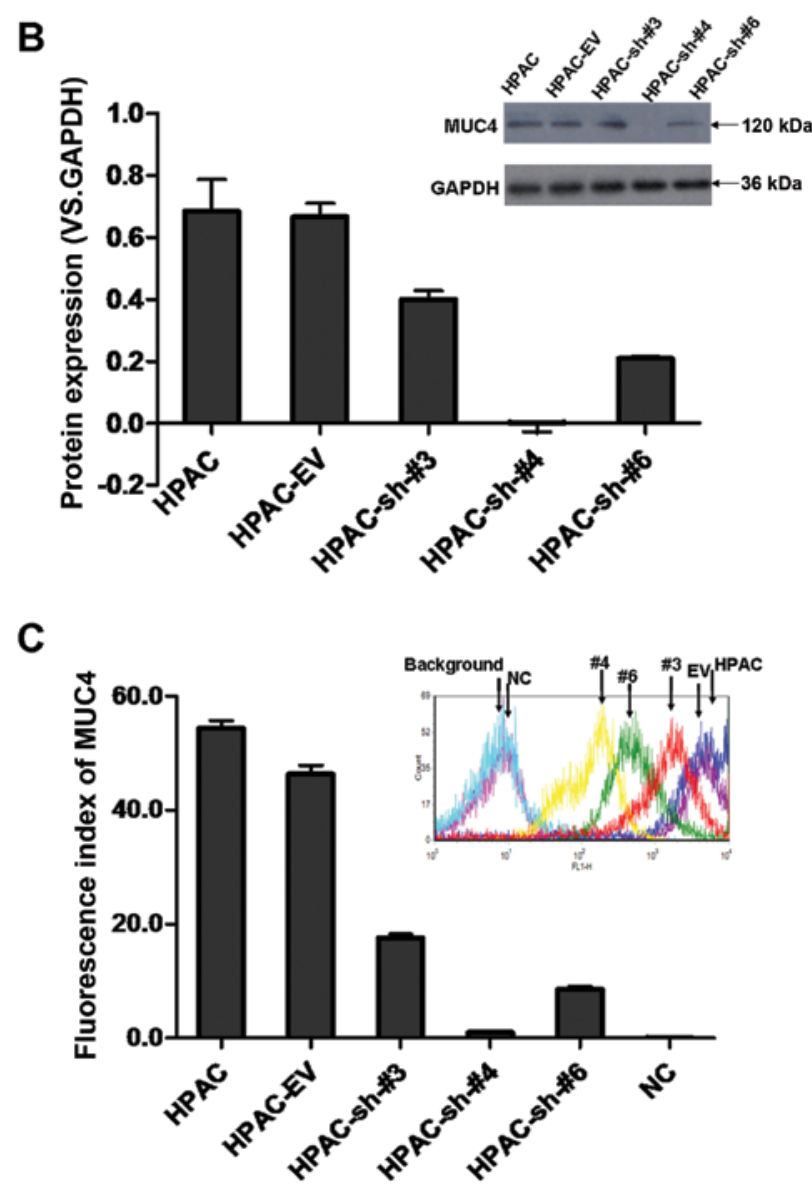

Figure 3. The selection of MUC4-shRNA HPAC sublines with different MUC4 expression. (A) Quantitative real-time PCR analysis of MUC4 mRNA expression in seven stably transfected clones. HPAC-EV represents transfected empty vector clone of HPAC. HPAC-sh-\#1-6, represent six stably transfected MUC4-shRNA clones of HPAC. (B) Western blot analysis and optical density (OD) analysis of the relative protein expression levels of MUC4 (vs. GAPDH) in HPAC-sh-\#3,4,6, respectively. Formula: (OD value of MUC4 - OD value of background) / (OD value of GAPDH - OD value of background). (C) FACS analysis of cell membrane surface expression of MUC4 in HPAC sublines. Fluorescence index (FI) was calculated with the following formula: FI = (GMFI sample - GMFI background) / GMFI background. GMFI represents the value of geometric mean fluorescence intensity. $\mathrm{NC}$, negative control. GMFI of equal quantity cells only labeled primary antibodies were calculated as background values. GMFI of equal quantity cells only labeled second antibodies were calculated as NC. All data are expressed as the means \pm standard deviation (SD) of three independent experiments.

tively. This is consistent with the results of the western blot analysis. These results demonstrated higher, medium and lower reduction of MUC4 expression in HPAC-sh-\#4, HPAC-sh-\#6 and HPAC-sh-\#3, and were therefore termed HPAC-sh-Higher 
A
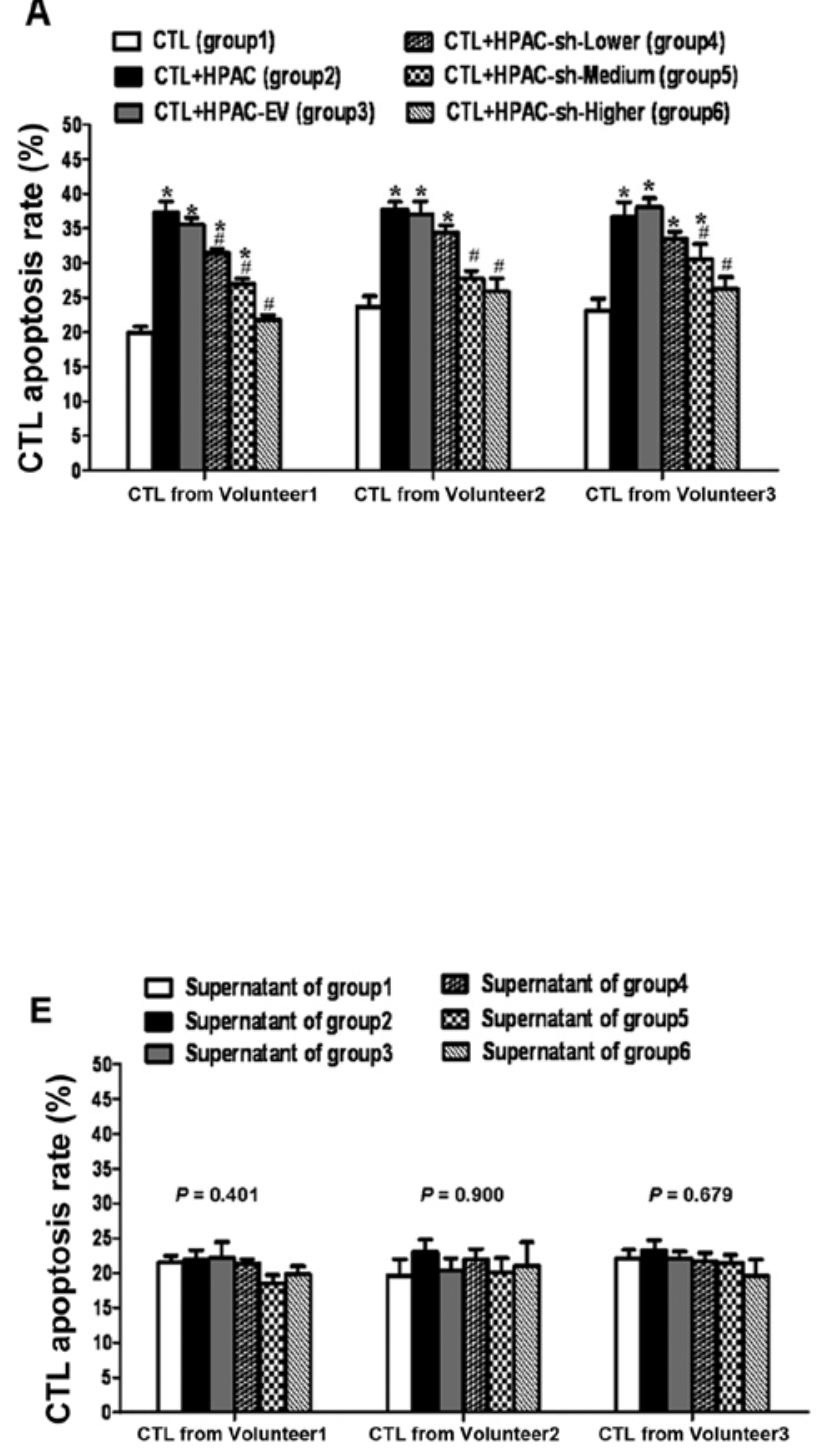

B

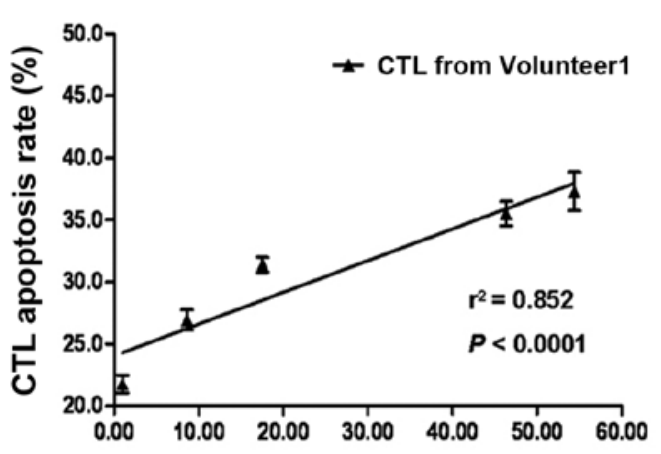

Surface expression levels of MUC4 on HPAC (MFI)

C

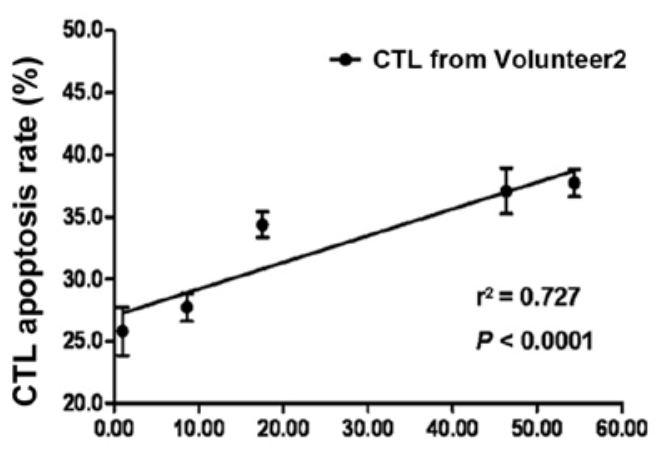

D

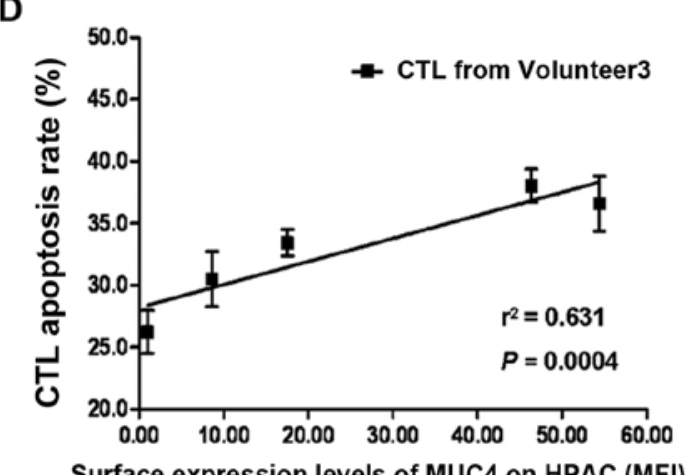

Surface expression levels of MUC4 on HPAC (MFI)

Figure 4. Effects of MUC4 expression on HPAC surface and supernatants on MS-CTL apoptosis in a co-incubation system. (A) Comparison of the level of MS-CTL apoptosis rate in different groups with HPAC sublines. ${ }^{*} \mathrm{P}<0.05$ in comparison with group $1,{ }^{*} \mathrm{P}<0.05$ in comparison with group 2. (B-D) Strong positive correlation between CTL apoptosis rate and the surface MUC4 expression levels of HPAC sublines. (E) The influence of the supernatants of group 1-6 on MS-CTL apoptosis was not significantly different $(\mathrm{P}>0.05)$. Error bars indicate the means \pm SEM of three determinations. One-way ANOVA plus Tukey's post hoc comparison was used to calculate the significant difference.

(H-sh-H), HPAC-sh-Medium (H-sh-M), HPAC-sh-Lower (H-sh-L), respectively.

MUC4 expression on HPAC surface contributes to MS-CTL apoptosis, while the influence of supernatants does not significantly correlate with MS-CTL apoptosis. Induction of MS-CTLs using blood sample from volunteer 1: compared to background control (group 1), the apoptosis rate of MS-CTLs in HPAC group (group 2), HPAC-EV group (group 3), H-sh-L group (group 4), and H-sh-M (group 5) increased significantly, $\mathrm{P}<0.05$, respectively (Fig. $4 \mathrm{~A}$ ). Compared to group 2, MS-CTL apoptosis rate in groups 4,5 and $\mathrm{H}$-sh-H (group 6) decreased significantly, $\mathrm{P}<0.05$, respectively (Fig. 4A). Meanwhile, as shown in Fig. 4B, a strong positive correlation $\left(r^{2}=0.852, P<0.0001\right)$ was observed between CTL apoptosis rate and the surface expression levels of MUC4 on
HPAC sublines indicated by mean fluorescence index (MFI). Using blood samples from volunteers 2 and 3 , the variation of data presented a similar general trend, although the changing extent was different (Fig. 4B and D). Therefore, these results demonstrated that surface expression of MUC4 on HPACs contributed to MS-CTL apoptosis.

After MS-CTLs were re-cultured using the supernatants of groups 1-6 with fixed basic condition, one-way ANOVA demonstrated MS-CTL apoptosis had no statistically significant differences $(\mathrm{P}>0.05)$ among distinct groups (Fig. 4E). The result showed that the total effects of supernatants which might hold various soluble factors were not clearly produced on MS-CTL apoptosis.

Fas-blockade has no significant effect on MUC4-mediated MS-CTL apoptosis. Although high levels of MUC4 expres- 

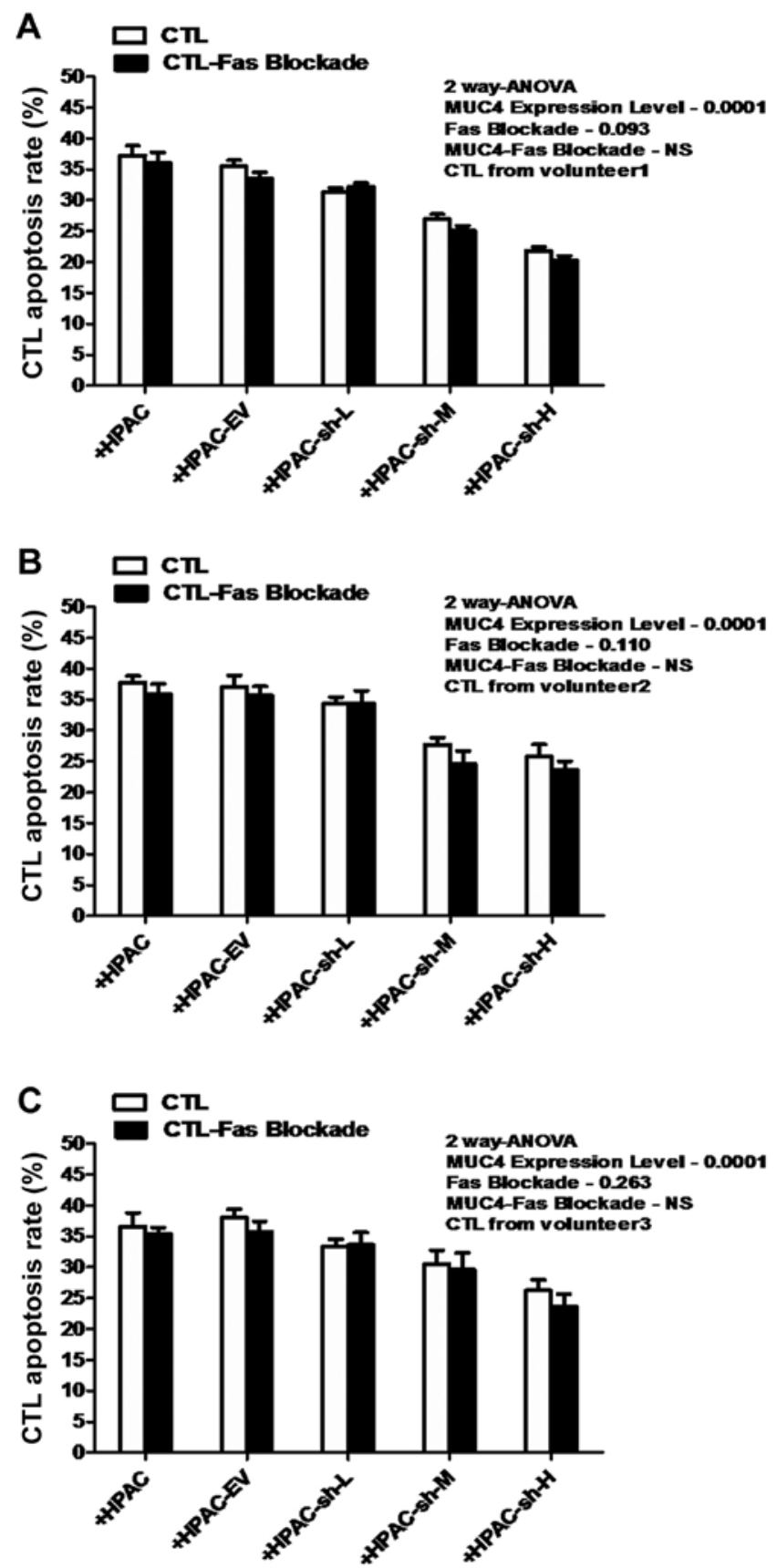

Figure 5. No significant effect of Fas-blockade/non on MUC4-mediated MS-CTL apoptosis. Two-way ANOVA indicated that Fas-blockade/non had no statistically significant effect on MS-CTL apoptosis $(\mathrm{P}>0.05)$. At the same time, the effect of interaction of the factors (Fas-blockade and MUC4expression) was non-significant (NS).

sion on HPAC surface were verified to have an effect on the significant increase of apoptosis of MS-CTLs during tumor cell-CTL interaction, whether the classical Fas-FasL pathway is actively involved in this process or not remains to be determined. As shown in Fig. 5, two-way ANOVA indicated that Fas-blockade/non had no statistically significant effect on MS-CTL apoptosis $(\mathrm{P}>0.05)$. At the same time, the effect of interaction of the factors (Fas-blockade and MUC4-expression) was non-significant. These results indicated that the Fas-FasL pathway did not participate in the process of MUC4-mediated MS-CTL apoptosis.
Collectively, the results suggested that during the cytolytic process, MUC4-bearing tumor cells could counterattack MS-CTLs via cell surface MUC4-mediated MS-CTL apoptosis, indicating a novel Fas-independent counterattack pathway, although the underlying mechanism may be diverse.

\section{Discussion}

As a class of molecules with significant roles in cell-cell communication, tumor-associated mucins, including human mucin4 (MUC4), might affect the immune response in several ways: they might provide an impenetrable barrier for immune effector cells, preventing an antitumor response; they might inactivate immune effector cells through receptor-ligand interactions; or they might sequester cytokines or other compounds that suppress a tumor immune response (19).

Although the exact mechanisms of immune regulation by MUC4 are not yet clear, our observations support the hypothesis that MUC4 may be employed by MUC4-bearing cancer cells to counterattack immune cells for their enhanced survival. Our data clearly indicate that the apoptosis of MS-CTLs can be mediated by membrane-associated MUC4 on the surface of pancreatic cancer cells during the cytolytic process. However, our data showed that supernatants (which could contain various soluble factors generated by the interaction of cancer cells and CTLs) have non-significant effects on the apoptosis of MS-CTLs. Moreover, in light of previous reports, three soluble factors, TNF- $\alpha$ (CD120a,b) $(26,27)$, TNF-related apoptosis-inducing ligand (TRAIL) $(28,29)$ and secreted forms of MUC4 (sMUC4) (30), could be involved in the apoptosis induction and function inhibition of activated $\mathrm{T}$ cells. We also quantitatively determined the levels of these three soluble apoptosis-related factors in supernatants with standard ELISA kits. The concentrations of three factors appeared to be in the picomolar or nanomolar ranges, respectively, but there was no significant difference in TNF- $\alpha$, TRAIL or sMUC4 levels among distinct groups and MS-CTL apoptosis did not correlate with these soluble factors (unpublished data). Thus, these two aspects clearly show that MUC4-mediated apoptosis of MS-CTLs is contact-dependent of effector cell with target cell.

Currently, it is considered that the Fas-FasL pathway can result in T-cell suicide and fratricide, known as activationinduced cell death (AICD) (31-33). In the present study, a small degree of apoptosis ( 20\%) of background control (CTLs without target cells added) was observed, which could occur related with that it took two weeks to induce MUC4 specific CTLs from peripheral blood in vitro and cell-culture medium containing IL-2 might induce the FasL expression on CTL surface and contribute to the AICD effect. FasL expression on tumor cells is considered the 'counterattack' of tumor to Fas-expressing effector $\mathrm{T}$ lymphocytes, which leads to the occurrence of immune evasion (33-35). However, our data demonstrated that blockade of Fas receptor by pretreatment with the antagonistic Fas antibody (ZB4) did not significantly inhibit the occurrence of MS-CTL apoptosis during the control experiments and the parallel processing systems, suggesting that mainly the cell death stimulus is initiated through a Fas-independent pathway; it might be possible that the interaction of MUC4 (functionally characterized domains) 


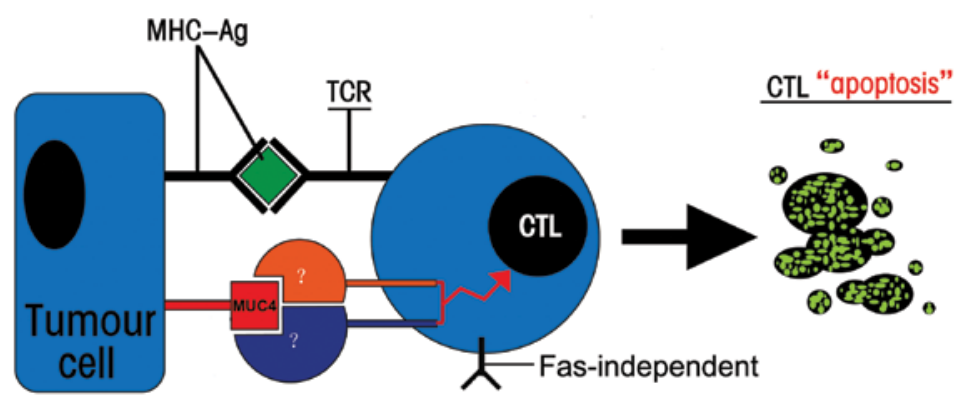

Figure 6. Schematic diagram of a novel counterattack pathway of MUC4-bearing tumor cells. It is a MUC4-mediated, cell contact-dependent and Fasindependent process to induce CTL apoptosis by initiating downstream signal transduction cascade.

with other receptors on $\mathrm{T}$ cells results in a series of apoptosis signal transductions.

Additionally, we utilized CTL self control group or CTL added with wild tumor cell group to rule out the possible effect of intrinsic activated cell-autonomous death (ACAD) (32) and analogous antigen-dependent apoptosis of CTL (ADAC) (36) in our study system, which gave indirect evidence to the localization of extrinsic MUC4-mediated apoptosis of MS-CTLs. Generally, the pathway of the T-cell apoptosis is considered the intrinsic and extrinsic apoptotic pathway, meanwhile, the absence of appropriate survival signals can cause ACAD (32). On the other hand, ADAC appears only when there is both excessive antigen density and a sufficiently high avidity in the TCR-MHC/peptide interaction (36). However, in the present study, MS-CTL apoptosis did not agree with ACAD and ADAC, since the induction process of MS-CTL is fixed and standard by using appropriate amounts of MUC4 epitope peptide. Moreover, the presence of extrinsic MUC4-mediated apoptosis of MS-CTLs can be verified by implementing the appropriate controls.

In addition, regulatory $\mathrm{T}$ cells (Tregs, $\mathrm{CD} 4^{+} \mathrm{CD} 25^{+}$) are considered to suppress effector T-cell activation, proliferation, and cytokine production $(37,38)$. In view of the fact that Treg cells are capable of killing autologous $\mathrm{CD}^{+} \mathrm{T}$ cells, $\mathrm{CD} 8^{+} \mathrm{T}$ lymphocytes were isolated from the PBMCs before the induction and amplification of MS-CTLs in order to eliminate the effect of Tregs in this study.

As shown in Fig. 6, the present study may provide a first step towards the elucidation of this mechanism in the apoptosis of MUC4-specific CTLs, suggesting that high levels of MUC4 molecules on cancer cell surface as a ligand might induce apoptosis of MS-CTLs by binding to undiscovered receptor on $\mathrm{T}$ cells to initiate a signal transduction cascade of apoptosis. Muc4/MUC4 was confirmed to be a ligand/ modulator for the receptor tyrosine kinase ErbB2, regulating its phosphorylation and the phosphorylation of its partner ErbB3, with or without the involvement of the ErbB3 ligand neuregulin, which induces signaling related to growth, motility, or differentiation properties of the cell (39-41). It has been speculated that the EGF-like domain of MUC4 specific domains plays a role in the above receptor-ligand interactions $(42,43)$. Meanwhile, the other unique domains present in the MUC4 mucin but not found in other membranebound mucins are NIDO, AMOP, and vWD domains (44). Although there is no direct evidence in the literature yet, the homology and evolution analysis hints at a role for both
NIDO and AMOP domain in cell-cell interaction and adhesion to the extracellular matrix (45-48). Whether MUC4 itself can establish novel interactions with receptor protein on the surface of CTLs via functionally characterized domain is yet to be confirmed. Further studies on the signaling pathways downstream of CTL apoptosis are presently being carried out by means of construction of functionally primed human $\mathrm{CD} 8^{+}$ MUC4-specific CTL clones model system, target molecule screening, domain deletion mutant, function-blocking and other techniques in our laboratory.

In summary, several lines of evidence suggest that there may be a novel counterattack pathway of pancreatic cancer cells, which is a MUC4-mediated, cell contact-dependent and Fas-independent process, to induce CTL apoptosis and attenuate the antitumor effect of CTLs (Fig. 6).

MUC4 is aberrantly expressed in a variety of human epithelial malignancies, particularly in pancreatic cancer (48), hence, the development and implications of anti-MUC4-specific immunotherapeutic strategies are important. Therefore, further exploration and understanding of the potential counterattack mechanisms and the intention to develop strategies to prevent or bypass apoptosis of CTLs is highly advantageous in enhancing the efficacy of MUC4-specific tumor vaccines.

\section{Acknowledgements}

The present study was partially supported by the National Natural Science Foundation of China (81101802, 81001079), the Natural Science Foundation of Jiangsu Province (BK2011845), the Program for Development of Innovative Research Team in the First Affiliated Hospital of NJMU, the Priority Academic Program Development of Jiangsu Higher Education Institutions (PAPD, JX10231801), and the Research Special Fund for Public Welfare Industry of Health (201202007).

\section{References}

1. Wong HH and Lemoine NR: Biological approaches to therapy of pancreatic cancer. Pancreatology 8: 431-461, 2008.

2. Li J, Merl MY, Chabot J and Saif MW: Updates of adjuvant therapy in pancreatic cancer: where are we and where are we going? Highlights from the ' 2010 ASCO Annual Meeting'. Chicago, IL, USA. June 4-8, 2010. JOP 11: 310-312, 2010.

3. Porchet N, Nguyen VC, Dufosse J, et al: Molecular cloning and chromosomal localization of a novel human tracheo-bronchial mucin cDNA containing tandemly repeated sequences of 48 base pairs. Biochem Biophys Res Commun 175: 414-422, 1991. 
4. Moniaux N, Nollet S, Porchet N, Degand P, Laine A and Aubert JP: Complete sequence of the human mucin MUC4: a putative cell membrane-associated mucin. Biochem J 338 : 325-333, 1999.

5. Rachagani S, Torres MP, Moniaux N and Batra SK: Current status of mucins in the diagnosis and therapy of cancer. Biofactors 35: 509-527, 2009

6. Torres MP, Chakraborty S, Souchek J and Batra SK: Mucinbased targeted pancreatic cancer therapy. Curr Pharm Des 18 2472-2481, 2012.

7. Andrianifahanana M, Moniaux N, Schmied BM, et al: Mucin $(M U C)$ gene expression in human pancreatic adenocarcinoma and chronic pancreatitis: a potential role of MUC4 as a tumor marker of diagnostic significance. Clin Cancer Res 7: 4033-4040, 2001.

8. Jhala N, Jhala D, Vickers SM, et al: Biomarkers in diagnosis of pancreatic carcinoma in fine-needle aspirates. Am J Clin Pathol 126: 572-579, 2006

9. Zhu Y, Zhang JJ, Zhu R, et al: The increase in the expression and hypomethylation of MUC4 gene with the progression of pancreatic ductal adenocarcinoma. Med Oncol 28 (Suppl 1): S175-S184, 2011.

10. Saitou M, Goto M, Horinouchi M, et al: MUC4 expression is a novel prognostic factor in patients with invasive ductal carcinoma of the pancreas. J Clin Pathol 58: 845-852, 2005

11. Bafna S, Kaur S, Momi N and Batra SK: Pancreatic cancer cells resistance to gemcitabine: the role of MUC4 mucin. Br J Cancer 101: 1155-1161, 2009.

12. Chaturvedi P, Singh AP, Moniaux N, et al: MUC4 mucin potentiates pancreatic tumor cell proliferation, survival, and invasive properties and interferes with its interaction to extracellular matrix proteins. Mol Cancer Res 5: 309-320, 2007.

13. Singh AP, Moniaux N, Chauhan SC, Meza JL and Batra SK: Inhibition of MUC4 expression suppresses pancreatic tumor cel growth and metastasis. Cancer Res 64: 622-630, 2004.

14. Rachagani S, Macha MA, Ponnusamy MP, et al: MUC4 potentiates invasion and metastasis of pancreatic cancer cells through stabilization of fibroblast growth factor receptor 1 . Carcinogenesis 33: 1953-1964, 2012.

15. Wei J, Gao W, Wu J, et al: Dendritic cells expressing a combined PADRE/MUC4-derived polyepitope DNA vaccine induce multiple cytotoxic T-cell responses. Cancer Biother Radiopharm 23: $121-128,2008$

16. Wu J, Wei J, Meng K, et al: Identification of an HLA-A*0201restrictive CTL epitope from MUC4 for applicable vaccine therapy. Immunopharmacol Immunotoxicol 31: 468-476, 2009.

17. Gao WT, Zhang JJ, Zhu Y, et al: Pentamer guided HLA-restricted epitope identification for mucoprotein 4 antigen of pancreatic ductal adenocarcinoma. Zhonghua Wai Ke Za Zhi 48: 1416-1424, 2010 (In Chinese).

18. Singh AP, Chaturvedi P and Batra SK: Emerging roles of MUC4 in cancer: a novel target for diagnosis and therapy. Cancer Res 67: 433-436, 2007.

19. Hollingsworth MA and Swanson BJ: Mucins in cancer: protection and control of the cell surface. Nat Rev Cancer 4: 45-60, 2004.

20. Qin Qin P, Su F, Xiao Yan W, et al: Distribution of human leucocyte antigen-A, -B and -DR alleles and haplotypes at high resolution in the population from Jiangsu province of China. Int J Immunogenet 38: 475-481, 2011.

21. Nagy P, Friedlander E, Tanner M, et al: Decreased accessibility and lack of activation of ErbB2 in JIMT-1, a herceptin-resistant, MUC4-expressing breast cancer cell line. Cancer Res 65: 473-482, 2005

22. Workman HC, Sweeney C and Carraway KL III: The membrane mucin Muc4 inhibits apoptosis induced by multiple insults via ErbB2-dependent and ErbB2-independent mechanisms. Cancer Res 69: 2845-2852, 2009

23. Moffat J, Grueneberg DA, Yang X, et al: A lentiviral RNAi library for human and mouse genes applied to an arrayed viral high-content screen. Cell 124: 1283-1298, 2006.

24. Ma C, Zhang J, Durrin LK, et al: The BCL2 major breakpoint region (mbr) regulates gene expression. Oncogene 26: 2649-2657, 2007.
25. Kim GG, Donnenberg VS, Donnenberg AD, Gooding W and Whiteside TL: A novel multiparametric flow cytometry-based cytotoxicity assay simultaneously immunophenotypes effector cells: comparisons to a $4 \mathrm{~h}{ }^{51} \mathrm{Cr}$-release assay. J Immunol Methods 325: 51-66, 2007

26. Sedgwick JD, Riminton DS, Cyster JG and Korner H: Tumor necrosis factor: a master-regulator of leukocyte movement. Immunol Today 21: 110-113, 2000.

27. Moss ML, Jin SL, Milla ME, et al: Cloning of a disintegrin metalloproteinase that processes precursor tumour-necrosis factor- $\alpha$. Nature 385: 733-736, 1997.

28. Wiley SR, Schooley K, Smolak PJ, et al: Identification and characterization of a new member of the TNF family that induces apoptosis. Immunity 3: 673-682, 1995.

29. Pitti RM, Marsters SA, Ruppert S, Donahue CJ, Moore A and Ashkenazi A: Induction of apoptosis by Apo-2 ligand, a new member of the tumor necrosis factor cytokine family. J Biol Chem 271: 12687-12690, 1996.

30. Komatsu M, Yee L and Carraway KL: Overexpression of sialomucin complex, a rat homologue of MUC4, inhibits tumor killing by lymphokine-activated killer cells. Cancer Res 59: 2229-2236, 1999.

31. Nagata S: Apoptosis by death factor. Cell 88: 355-365, 1997.

32. Krammer PH, Arnold R and Lavrik IN: Life and death in peripheral T cells. Nat Rev Immunol 7: 532-542, 2007.

33. Maher S, Toomey D, Condron C and Bouchier-Hayes D: Activation-induced cell death: the controversial role of Fas and Fas ligand in immune privilege and tumour counterattack. Immunol Cell Biol 80: 131-137, 2002.

34. Rivoltini L, Carrabba M, Huber V, et al: Immunity to cancer: attack and escape in $\mathrm{T}$ lymphocyte-tumor cell interaction. Immunol Rev 188: 97-113, 2002.

35. Igney FH, Behrens CK and Krammer PH: CD95L mediates tumor counterattack in vitro but induces neutrophil-independent tumor rejection in vivo. Int J Cancer 113: 78-87, 2005.

36. Derby MA, Snyder JT, Tse R, Alexander-Miller MA and Berzofsky JA: An abrupt and concordant initiation of apoptosis: antigen-dependent death of CD8 ${ }^{+}$CTL. Eur J Immunol 31: 2951-2959, 2001

37. Khazaie $\mathrm{K}$ and von Boehmer $\mathrm{H}$ : The impact of $\mathrm{CD} 4^{+} \mathrm{CD} 25^{+}$Treg on tumor specific $\mathrm{CD}^{+} \mathrm{T}$ cell cytotoxicity and cancer. Semin Cancer Biol 16: 124-136, 2006.

38. Miyara M and Sakaguchi S: Natural regulatory T cells: mechanisms of suppression. Trends Mol Med 13: 108-116, 2007.

39. Carraway KL, Perez A, Idris N, et al: Muc4/sialomucin complex, the intramembrane ErbB2 ligand, in cancer and epithelia: to protect and to survive. Prog Nucleic Acid Res Mol Biol 71: $149-185,2002$

40. Jepson S, Komatsu M, Haq B, et al: Muc4/sialomucin complex, the intramembrane ErbB2 ligand, induces specific phosphorylation of ErbB2 and enhances expression of $\mathrm{p} 27^{\mathrm{kip}}$, but does not activate mitogen-activated kinase or protein kinaseB/Akt pathways. Oncogene 21: 7524-7532, 2002.

41. Chaturvedi P, Singh AP, Chakraborty S, et al: MUC4 mucin interacts with and stabilizes the HER 2 oncoprotein in human pancreatic cancer cells. Cancer Res 68: 2065-2070, 2008.

42. Carraway KL, Ramsauer VP, Haq B and Carothers Carraway CA: Cell signaling through membrane mucins. Bioessays 25: 66-71, 2003.

43. Carraway CA and Carraway KL: Sequestration and segregation of receptor kinases in epithelial cells: implications for ErbB2 oncogenesis. Sci STKE 2007: re3, 2007.

44. Desseyn JL, Tetaert D and Gouyer V: Architecture of the large membrane-bound mucins. Gene 410: 215-222, 2008.

45. Timpl R: Structure and biological activity of basement membrane proteins. Eur J Biochem 180: 487-502, 1989.

46. Mayer U, Kohfeldt E and Timpl R: Structural and genetic analysis of laminin-nidogen interaction. Ann NY Acad Sci 857: 130-142, 1998.

47. Ciccarelli FD, Doerks T and Bork P: AMOP, a protein module alternatively spliced in cancer cells. Trends Biochem Sci 27: $113-115,2002$.

48. Chaturvedi P, Singh AP and Batra SK: Structure, evolution, and biology of the MUC4 mucin. FASEB J 22: 966-981, 2008. 